\title{
EVALUACIÓN DEL IMPACTO AMBIENTAL AL EXTENDER LA VIDA ÚTIL DEL TELÉFONO MÓVIL
}

\author{
Samantha Eugenia CRUZ SOTELO ${ }^{1 *}$, María Dolores BOVEA EDO ${ }^{2}$, Sara OJEDA BENITEZ ${ }^{3}$ \\ Néstor SANTILLÁN SOTO ${ }^{3}$ y Onofre Rafael GARCÍA CUETO ${ }^{3}$
}

${ }^{1}$ Facultad de Ingeniería, Universidad Autónoma de Baja California. Bulevar Benito Juárez y calle de la Normal, Insurgentes Este, Mexicali, Baja California, México, C. P. 21280

${ }^{2}$ Departamento de Ingeniería Mecánica y Construcción, Universitat Jaume I. Avenida Sos Baynat, E-12071, Castellón de la Plana, España, C. P. 12006

${ }^{3}$ Instituto de Ingeniería, Universidad Autónoma de Baja California. Bulevar Benito Juárez y calle de la Normal, Insurgentes Este, Mexicali, Baja California, México, C. P. 21280

*Autor para correspondencia: samantha.cruz@uabc.edu.mx

(Recibido septiembre 2016; aceptado diciembre 2016)

Palabras clave: análisis de ciclo de vida, escenarios, inventario, valorización

\section{RESUMEN}

El mercado de telefonía móvil ha experimentado un continuo crecimiento en la última década. Cada vez más, los teléfonos móviles son reemplazados por nuevos modelos con diseños más modernos y ampliación de funciones. Este estilo de vida está provocando incremento en la cantidad de residuos de aparatos electrónicos que no siempre reciben tratamiento. Por lo que, en este artículo, se presentan los resultados de la aplicación de la metodología de análisis de ciclo de vida (ACV) para calcular cuantitativamente el beneficio ambiental debido a la extensión de vida útil del teléfono móvil durante diferentes períodos. Tomando como unidad funcional el ciclo de vida promedio de 30 meses, se modelaron tres escenarios considerando cuatro etapas: adquisición de materias primas/producción, distribución, uso y fin de vida útil. Se utilizó el paquete SimaPro (2013), la base de datos Ecoinvent para configurar el inventario y las emisiones consideradas se asignaron en seis categorías de impacto según el método CML2000 del Centro para Estudios Medioambientales (CML). Se observó que al prolongar la vida útil del teléfono móvil y dar tratamiento cuando se convierte en residuo representa una ganancia ambiental no sólo porque se evita su disposición y consumo de uno nuevo, sino porque se ahorran recursos limitados y la energía necesaria para su extracción a través de la recuperación de materiales. Sin embargo, antes de reciclar es importante considerar su reutilización (extensión de su vida útil) ya que una cantidad sustancial de los móviles son dispuestos por razones de moda o estética mientras aún son funcionales.

Key words: life cycle assessment, scenarios, inventory, valorization

\begin{abstract}
The mobile phone market has experienced continuous growth over the last decade. Increasingly, mobile phones are replaced by new models with more modern designs or expansion of functions. This lifestyle is causing increasing amount of electronic waste
\end{abstract}


that do not always receive treatment. This article presents the results of the application of the methodology for life-cycle assessment (LCA) to calculate quantitatively the environmental benefit due to the extension of lifespan mobile phones during different periods of time. Taking as a functional unit the life cycle of an average mobile phone during 30 months and considering the stages of raw materials/production, distribution, use and end of life, three scenarios were modeled. The SimaPro (2013) software was used, the Ecoinvent database has been applied to configure the life cycle inventory, and the emissions were allocated into six categories according to the impact CML2000 method. It was observed that to extend the mobile phone useful life and to provide treatment when it becomes a waste, represents an environmental gain not only because the provision and consumption of a new one is avoided, but because limited resources and energy required for extraction are saved through recovery of materials. However, before recycling it is important to consider reuse (extending lifespan) because a substantial amount of the mobile phones are discarded for fashion or cosmetic reasons while they are still functional.

\section{INTRODUCCIÓN}

La producción de aparatos eléctricos y electrónicos (AEE) es uno de los sectores de mayor crecimiento de la industria manufacturera en el mundo (Widmer 2005, Peralta 2006). Por otra parte, la industria de los AEE es la responsable de entre el 10 y $20 \%$ del impacto ambiental global en relación con el agotamiento de los recursos no renovables (Kahan 2016).

Los residuos de aparatos eléctricos y electrónicos (RAEE) han sido motivo de interés en las últimas décadas porque tiene un flujo complejo por su composición y con un crecimiento acelerado. Cucchiella et al. (2015), mencionan que el flujo de RAEE aumenta más rápido que el resto de los residuos, se estima que crece entre 3 y $5 \%$ anualmente. De acuerdo con un reporte de la Universidad de las Naciones Unidas en 2014 se generaron, en el mundo, cerca de 42 millones de toneladas de RAEE (Baldé et al. 2015). México se encuentra entre los 40 países del mundo y seis latinoamericanos que desechan más aparatos electrónicos (Magaline et al. 2015).

Esta tendencia representa problemas ambientales y aumenta la necesidad de implementar acciones para su gestión (Mohabuth y Miles 2005, Tartiu 2009, Song et al. 2013), en la búsqueda de la minimización de impactos y de medidas para la valorización y aprovechamiento de los recursos (Ylä-Mella 2004, Sinha et al. 2005).

La importancia en la gestión de los RAEE se debe a las particularidades que los diferencian de otros tipos de residuos. De acuerdo con su composición pueden manejarse como residuos peligrosos o de manejo especial. Sin embargo, considerando la fuente de generación es común que sean manejados con el flujo de los residuos sólidos urbanos (RSU).
En cuanto a la electrónica de consumo, las tecnologías de la información y la comunicación (TIC) son un sector que ha estado creciendo muy rápido, entre los dispositivos de este sector están los teléfonos móviles (Aleksic 2014). La introducción de nuevas tecnologías, con más aplicaciones y más funcionales provoca que los ciclos de vida de los dispositivos sean relativamente cortos, convirtiéndose rápidamente en tecnología obsoleta (Osibanjo y Nnorom 2008, Nnorom et al. 2009, Kasper et al. 2011, Kiddee et al. 2013, Suckling y Lee 2015).

La tecnología móvil es un mercado de rápido crecimiento, el uso del teléfono móvil ha crecido de manera exponencial pasando de 4.7 billones de suscriptores en el mundo en 2009, a 7.2 billones de suscriptores en 2015, aumentado el número de suscriptores $39 \%$, en países desarrollados y $249 \%$ en países en desarrollo en un periodo de 10 años. En el mundo la tasa de penetración de la telefonía móvil ascendió a 97 \% (ITU 2016, Welfens et al. 2016) creando al mismo tiempo problemas de generación de residuos de teléfonos móviles y la necesidad de su gestión.

Para la gestión ambiental, existen metodologías científicas que evalúan productos, procesos o servicios, entre ellas está el análisis del ciclo de vida (ACV) (ISO 140402006 e ISO14044 2006). El ACV se ha aplicado para comparar sistemas de manejo y evaluar las cargas ambientales asociadas, identificando y cuantificando el uso de materia, energía y los vertidos al entorno para determinar las consecuencias que su uso produce en el ambiente (Barton et al. 1996, Ekvall et al. 2007, Obersteiner et al. 2007, Schmidt y Pahl-Wostl 2007, Winkler y Bilitewski 2007).

Esta metodología también se ha aplicado a etapas de gestión de residuos sólidos urbanos, tales como 
transporte, disposición y aprovechamiento (Foolmaun y Ramjeawon 2008, Cleary 2009, Rives et al. 2010). Asimismo, para la evaluación de los impactos que provoca la producción del teléfono móvil (Yamaguchi et al. 2003), el consumo de materiales y la energía durante su ciclo de vida (Yu et al. 2010).

El propósito de esta investigación fue calcular el beneficio ambiental al extender la vida útil del teléfono móvil en las etapas de adquisición de materias primas/producción, distribución, uso y fin de vida útil con el método del ACV en tres escenarios. Se entiende por vida útil, el periodo de tiempo estimado que el teléfono móvil puede tener para cumplir correctamente con su función. En el caso que abordamos, la vida útil está asociada con la obsolescencia que determina el usuario.

\section{MATERIALES Y MÉTODOS}

Para realizar esta investigación se aplicó el método de $\mathrm{ACV}$, siguiendo las cuatro etapas de acuerdo con la normas ISO 14040 (2006) e ISO 14044 (2006): definición de objetivos y alcance, análisis de inventario, evaluación e Interpretación de impactos.

\section{Definición de objetivo y alcance}

Para aplicar la metodología a este estudio de caso se asumió como escenario de referencia el tiempo promedio de uso de un teléfono móvil reportado por Cruz (2103), agregando seis meses a cada escenario propuesto, porque es el periodo de tiempo en el que se observan diferencias en el tiempo de vida útil del teléfono móvil, de acuerdo al usuario.

Se evaluó el impacto ambiental conforme a tres escenarios de manejo del teléfono móvil, el criterio de análisis fue el promedio de tiempo de uso. El escenario base fue el uso del teléfono móvil durante 18 meses (situación actual, $\mathrm{S}_{0}$ ), la extensión de su vida hasta 24 meses (escenario $1, \mathrm{~S}_{1}$ ) y 30 meses (escenario 2, $\mathrm{S}_{2}$ ).

Posteriormente se determinó la unidad funcional (UF), definida como el uso y gestión del teléfono móvil durante 30 meses en tres escenarios, diferenciado por la duración de la vida útil del teléfono móvil (Fig. 1).

El escenario $\mathrm{S}_{0}$ requiere el uso de 1.66 teléfonos móviles de 18 meses de vida útil para alcanzar la UF, el escenario $S_{1}$ requiere de 1.25 teléfonos de 24 meses de vida útil, y el $S_{2}$ permite alcanzar con un único teléfono móvil la unidad funcional. Para cada uno de estos escenarios se consideraron las siguientes etapas del ciclo de vida de un teléfono móvil:

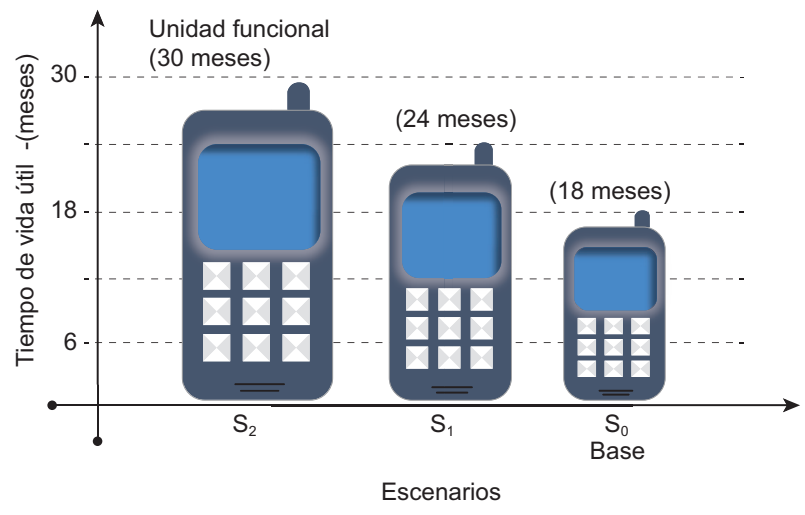

Fig. 1. Escenarios propuestos para el análisis de ciclo de vida Fuente. Elaboración propia

a) Etapa de adquisición/producción, se incluyen los porcentajes de componentes obtenidos mediante el análisis de los datos recolectados a través de la caracterización de 15 teléfonos móviles.

b) Etapa de distribución, corresponde a la producción del teléfono móvil en China y su posterior distribución desde la ciudad de México, por lo que se ha incluido el transporte en barco transoceánico (puerto Shanghái, China-Ensenada, México) y transporte terrestre (Ensenada-Ciudad de MéxicoMexicali).

c) Etapa de uso, para esta etapa se analizó información obtenida de la literatura para calcular la media de consumo energético de un móvil en su etapa de uso. Se multiplicó la media de consumo por $1.5\left(\right.$ para $\mathrm{S}_{0}$ ), por $2\left(\right.$ para $\mathrm{S}_{1}$ ) y por 2.5 (para $\mathrm{S}_{2}$ ), asumiendo que a mayor vida útil del teléfono móvil, menor tiempo dura la batería con carga, por lo que se recarga con mayor frecuencia.

d) Etapa de fin de vida, se incluye el transporte a la planta de tratamiento para el desmontaje y posterior reciclado de componentes y el propio proceso de separación y reciclaje con las cargas evitadas correspondientes. En cuanto al transporte, se considera el impacto por el transporte de Mexicali, Baja California, México a la planta de tratamiento de RAEE ubicada en Monterrey, Nuevo León, México. Para el proceso de tratamiento se considera la descontaminación y separación manual de componentes, quedando tres fracciones de residuos [placa de circuito impreso (PCI), pantalla de cristal líquido (LCD, por sus siglas en inglés), residuos y baterías], los cuales siguen distintos tratamientos para la recuperación de materiales.

Una vez definidos los escenarios a analizar se establecieron criterios de entrada por etapa de análisis de acuerdo con el cuadro I. 
CUADRO I. FACTORES POR ETAPA DEL ANALISIS DE CICLO DE VIDA

\begin{tabular}{lllll}
\hline Etapas de análisis & \multicolumn{4}{c}{ Escenarios de vida } \\
\cline { 2 - 5 } & & $\mathrm{S}_{0}$ & $\mathrm{~S}_{1}$ & $\mathrm{~S}_{2}$ \\
\hline Adquisición/producción & 1.66 & 1.25 & 1 & \\
Distribución & 1.66 & 1.25 & 1 & 1.2 \\
Uso & 1 & 1.1 & 1 \\
Fin de vida/reciclaje & 1.66 & 1.25 & 1 \\
\hline
\end{tabular}

$\mathrm{S}_{0}=$ escenario base (18 meses de uso), $\mathrm{S}_{1}=24$ meses de uso, $\mathrm{S}_{2}=30$ meses de uso

\section{Inventario de ciclo de vida (ICV)}

Para la adquisición de los datos del ICV, considerando su importancia en cuanto a disponibilidad, fiabilidad y calidad, se realizó la caracterización del teléfono móvil, se usaron fuentes secundarias de información tales como bases de datos, documentos oficiales y referencias científicas. Se modelaron tres escenarios de acuerdo con los supuestos que se presentan en el cuadro II.

Se utilizó la base de datos Ecoinvent (2008), para configurar el ICV, adaptando algunos datos al caso de estudio, tales como la aportación energética por tecnologías, el transporte, las distancias y el tratamiento de las fracciones separadas.

Una vez identificados los materiales y procesos de fabricación del teléfono móvil, se aplicaron los siguientes criterios en la definición del inventario:

a) Se tomó información de la base de datos Ecoinvent (2008), previa adaptación al caso de estudio situado en México (aportación energética por tecnologías, distancias y medios de transporte).

b) Se consideró la adquisición de materias primas, fabricación de componentes que forman parte del producto y el transporte de materiales de fabricación de acuerdo con consumos en China.

c) No se consideró el embalaje del producto terminado.

d) Se consideró la distribución del producto terminado (China-México).

e) Se consideró una eficiencia de reciclaje 1:1 de los materiales.

\section{Etapa 1: Adquisición/producción. Consideracio- nes y datos de entrada}

A partir de la caracterización del teléfono móvil promedio, se modelaron los resultados en el programa SimaPro. Los datos de inventario correspondientes a la etapa de adquisición de materia prima y producción se adaptaron de Ecoinvent (2008) según lo indicado anteriormente, asumiendo las siguientes características para cada componente de acuerdo con el cuadro III.

Cada teléfono móvil analizado tenía componentes y materiales propios, por lo que en lugar de obtener un inventario promedio, se modeló cada móvil en el paquete SimaPro y se obtuvieron los indicadores ambientales. Posteriormente se obtuvieron los indicadores promedio, que fueron modelados como representativos del teléfono móvil promedio durante las etapas de adquisición de materias primas y producción. Los datos que representan la etapa de adquisición/producción del ACV se muestran en el cuadro VI. Las emisiones consideradas para la fase de inventario se han asignado en seis categorías de impacto según el método CML (Guinée 2002).

\section{Etapa 2: Distribución. Consideraciones y datos de entrada \\ El cuadro VII presenta los recorridos considera- dos para el transporte del teléfono móvil del lugar de fabricación al distribuidor.}

CUADRO II. CRITERIOS DE ENTRADA POR ETAPA DE CICLO DE VIDA DEL PRODUCTO

\begin{tabular}{ll}
\hline Etapa & Información \\
\hline $\begin{array}{l}\text { Adquisición de materias } \\
\text { primas y producción }\end{array}$ & $\begin{array}{l}\text { Con base en la caracterización del teléfono móvil se obtuvo la composición media siguiente (peso } \\
\text { promedio } 91.97 \mathrm{~g}) \text { : plástico } 39.96 \% \text {, ferrosos } 2.41 \% \text {, metales no ferrosos } 5.04 \% \text {, PCI (placa de } \\
\text { circuito impreso) } 26.90 \% \text { y batería } 25.69 \% .\end{array}$ \\
\hline Distribución & $\begin{array}{l}\text { Suponiendo la producción del teléfono móvil en China y su transporte a México, se han considerado } \\
\text { los siguientes trayectos: } 10771 \text { kilómetros por barco transoceánico y } 2888 \mathrm{~km} \text { por carretera. }\end{array}$ \\
\hline Uso & $\begin{array}{l}\text { Los tres escenarios fueron analizados considerando un consumo energético promedio de } 9 \mathrm{kw} / \mathrm{h} / \text { año } \\
\text { (Rosen y Meier } 2001) \text { y utilizando la combinación de tecnología para la generación eléctrica en }\end{array}$ \\
& $\begin{array}{l}\text { México correspondiente al año 2012. El escenario base }\left(\mathrm{S}_{0}\right) \text { considera el reemplazo del teléfono } \\
\text { móvil a los } 18 \text { meses, } \mathrm{S}_{1} \text { asume la sustitución a los } 24 \text { meses, mientras que } \mathrm{S}_{2} \text { a los } 30 \text { meses. }\end{array}$ \\
\hline Fin de vida & $\begin{array}{l}\text { Se asume el reciclaje de las fracciones de residuos generados siguiente: plástico } 21.53 \%, \text { ferroso } \\
12.86 \% \text { y no ferrosos } 9.77 \% .\end{array}$ \\
\hline
\end{tabular}


CUADRO III. MATERIALES CONTENIDOS EN LOS COMPONENTES DEL TELÉFONO MÓVIL

\begin{tabular}{ll}
\hline Componente & Materiales \\
\hline Carcasas exteriores & $\begin{array}{l}\text { ABS (acrilonitrilo butadieno estireno) y PC (policarbonato) generalmente. Como consecuencia } \\
\text { del brillo y luminosidad de las piezas, se ha considerado una proporción de 20 \% de ABS y } \\
80 \% \text { de PC para las piezas inyectadas. También se emplea PS (poliestireno), PET (polietileno } \\
\text { tereftalato) y PP (polipropileno) en alguna de las piezas plásticas. }\end{array}$ \\
\hline Aleaciones de piezas metálicas: & Se considera el Zamak, con una composición de zinc, aluminio, cobre y magnesio (cuadro IV). \\
\hline Tornillos & Acero y latón en alguno de los casos. \\
\hline Teclados & Silicona como material principal, presentando alguna de ellas partes fabricadas en PC. \\
\hline Conectores & Cobre y caucho sintético. \\
\hline Componentes eléctricos/ & $\begin{array}{l}\text { La información se tomó de la base de datos Ecoinvent (2008), referente a "Life Cycle Inventories } \\
\text { of Electric and Electronic Equipment: Production, Use and Disposal", selecionando los } \\
\text { dispositivos más adecuados, creando y aproximando la composición de aquéllos que no se } \\
\text { encuentran incluidos en el manual, como el bocina, cámara, micrófono y antena (cuadro V). }\end{array}$ \\
\hline
\end{tabular}

CUADRO IV. PIEZA METÁLICA DE ALEACIÓN DE ZINC, ALUMINIO, COBRE Y MAGNESIO (ZAMAK)

\begin{tabular}{lc}
\hline Piezas ZAMAK & $\%$ \\
\hline Zinc & 92.33 \\
Aluminio & 6 \\
Cobre & 1.60 \\
Magnesio & 0.07 \\
\hline
\end{tabular}

CUADRO V. MÓDULO APROXIMADO DE COMPOSICIÓN DE COMPONENTES

\begin{tabular}{lcllllll}
\hline Bocina & $\%$ & Cámara & $\%$ & Micrófono & $\%$ & Antena & $\%$ \\
\hline Aluminio & 10 & Cobre & 25 & Aluminio & 70 & Latón & 20 \\
Hierro & 40 & Policarbonato & 25 & Cobre & 10 & Polibutadieno & 80 \\
Cobre & 9.9 & Cristal & 50 & Acero & 20 & & \\
Acero prensado & 25 & & & & & & \\
Espuma Poliuretano & 0.1 & & & & & & \\
ABS & 15 & & & & & & \\
\hline
\end{tabular}

CUADRO VI. ANÁLISIS DE CICLO DE VIDA DE UN TELÉFONO MÓVIL PROMEDIO

\begin{tabular}{ll}
\hline Contaminante & Contribución \\
\hline Antimonio, en tierra (recurso) & $4.88 \mathrm{E}-02 \mathrm{Kg} \mathrm{Sb}_{\mathrm{eq}}$ \\
Dióxido de carbono (emisiones aire) & $6.92 \mathrm{E}+00 \mathrm{Kg} \mathrm{CO}_{2}$ eq \\
Dióxido sulfuroso (emisiones aire) & $6.64 \mathrm{E}-02 \mathrm{Kg} \mathrm{SO}_{2}$ eq \\
Metano, trichlorofluoro-, CFC-11 (emisiones aire) & $8.10 \mathrm{E}-06 \mathrm{Kg} \mathrm{CFC}^{-11}$ eq \\
Eteno (emisiones aire) & $3.10 \mathrm{E}-03 \mathrm{Kg} \mathrm{C}_{2} \mathrm{H}_{4}$-eq \\
Fosfatos (emisiones agua) & $6.77 \mathrm{E}-03 \mathrm{Kg} \mathrm{PO}_{4}^{----e q}$ \\
\hline
\end{tabular}

$\mathrm{CFC}=$ clorofluorocarburos 
CUADRO VII. DISTRIBUCIÓN DE UN TELÉFONO MÓVIL PROMEDIO

\begin{tabular}{llrl}
\hline Etapa & Trayecto & $\mathrm{km}$ & Transporte \\
\hline \multirow{2}{*}{ Distribución } & Shangai, China-Ensenada, México & 10771 & Barco transoceánico \\
& Ensenada-México-Mexicali & 5589 & Terrestre \\
\hline
\end{tabular}

CUADRO VIII. COMBINACIÓN ENERGÉTICAEN MÉXICO, PARA EL AÑO 2013-2014 (SENER 2014).

\begin{tabular}{lclc}
\hline Tecnología de generación & $(\%)$ & Tecnología de generación & $(\%)$ \\
\hline Termoeléctrica & 19.16 & Nucleoeléctrica & 4.74 \\
Ciclo combinado & 50.4 & Eólica & 0.75 \\
Dual & 6.21 & Hidroeléctrica & 12.34 \\
Carboeléctrica & 6.40 & Fotovoltaica & 0.005 \\
\hline
\end{tabular}

Etapa 3: Uso: Consideraciones y datos de entrada

Para esta etapa se calculó la potencia media de consumo energético del móvil para los tres escenarios. De acuerdo con Rosen y Meier (2001), el consumo de un teléfono móvil es de aproximadamente $9 \mathrm{kw} / \mathrm{h} / \mathrm{año}$. Se consideró la aportación energética por tecnología, correspondiente a México (cuadro VIII).

Al tomar en cuenta $\mathrm{S}_{2}$ como UF, para este estudio se asumió un incremento del $10 \%$ para el $\left(S_{1}\right)$ y $20 \%$ para el $\left(S_{2}\right)$ en pérdida de eficiencia de la batería quedando las proporciones de consumo energético para cada escenario como se muestra en el cuadro IX.

CUADRO IX. FACTORES DE CONSUMO ENERGÉTICO POR ESCENARIO DE CICLO DE VIDA

\begin{tabular}{lccc}
\hline Variable & \multicolumn{3}{c}{ Escenarios } \\
\hline Consumo $(\mathrm{kw} / \mathrm{h})$ & $\mathrm{S}_{0}$ & $\mathrm{~S}_{1}$ & $\mathrm{~S}_{2}$ \\
& $(18$ meses $)$ & $(24$ meses $)$ & $(30$ meses $)$ \\
& 1.5 & 2 & 2.5 \\
\hline
\end{tabular}

Etapa 4: Fin de vida/Reciclaje. Consideraciones y datos de entrada

En la etapa de fin de vida, o desuso del teléfono móvil se incluyó el escenario de reciclaje. La figura 2 muestra los primeros tres materiales (PCI-LCD, residuo y baterías) obtenidos a través de un proceso de descontaminación y separación manual. Estos materiales pasan por procesos y tratamiento distintos para la recuperación de materiales. Además, se pueden observar los requerimientos para cada proceso así como los materiales recuperados.
En el cuadro $\mathbf{X}$ se presentan los componentes del teléfono móvil potencialmente reciclables y las entradas requeridas para cada proceso.

El cuadro XI muestra una relación de materiales primarios y secundarios. La columna de cargas evitadas representa los recursos o materias primas que se evita extraer de la naturaleza a través del uso del material secundario o reciclado. En la columna de materiales secundarios se observan los materiales reciclados que sustituyen el recurso primario.

Se asume que el tratamiento de los componentes del móvil es en la ciudad de Monterrey, Nuevo León, por lo que se considera el transporte del material de Mexicali a esta ciudad (2240 km transporte terrestre).

\section{Evaluación e interpretación de impactos}

La evaluación del impacto es un proceso técnico, cuantitativo y cualitativo para caracterizar y evaluar los efectos de los contaminantes en los ecosistemas, identificados en la etapa de inventario.

En esta etapa se cuantifican las alteraciones provocadas al ambiente derivadas de la acción humana a través de la ejecución de prácticas que demandan el consumo de materias y energía.

\section{RESULTADOS Y DISCUSIÓN}

En esta sección se presentan los resultados y se discuten los hallazgos de la aplicación de la metodología del ACV a la gestión del teléfono móvil por etapa y componente, comparando los tres escenarios propuestos.

El cuadro XII resume los resultados del ACV por escenario para cada categoría de impacto. Se observa que el escenario base $\mathrm{S}_{0}$ es el que ocasiona mayor impacto en todas las categorías, esto se atribuye al 


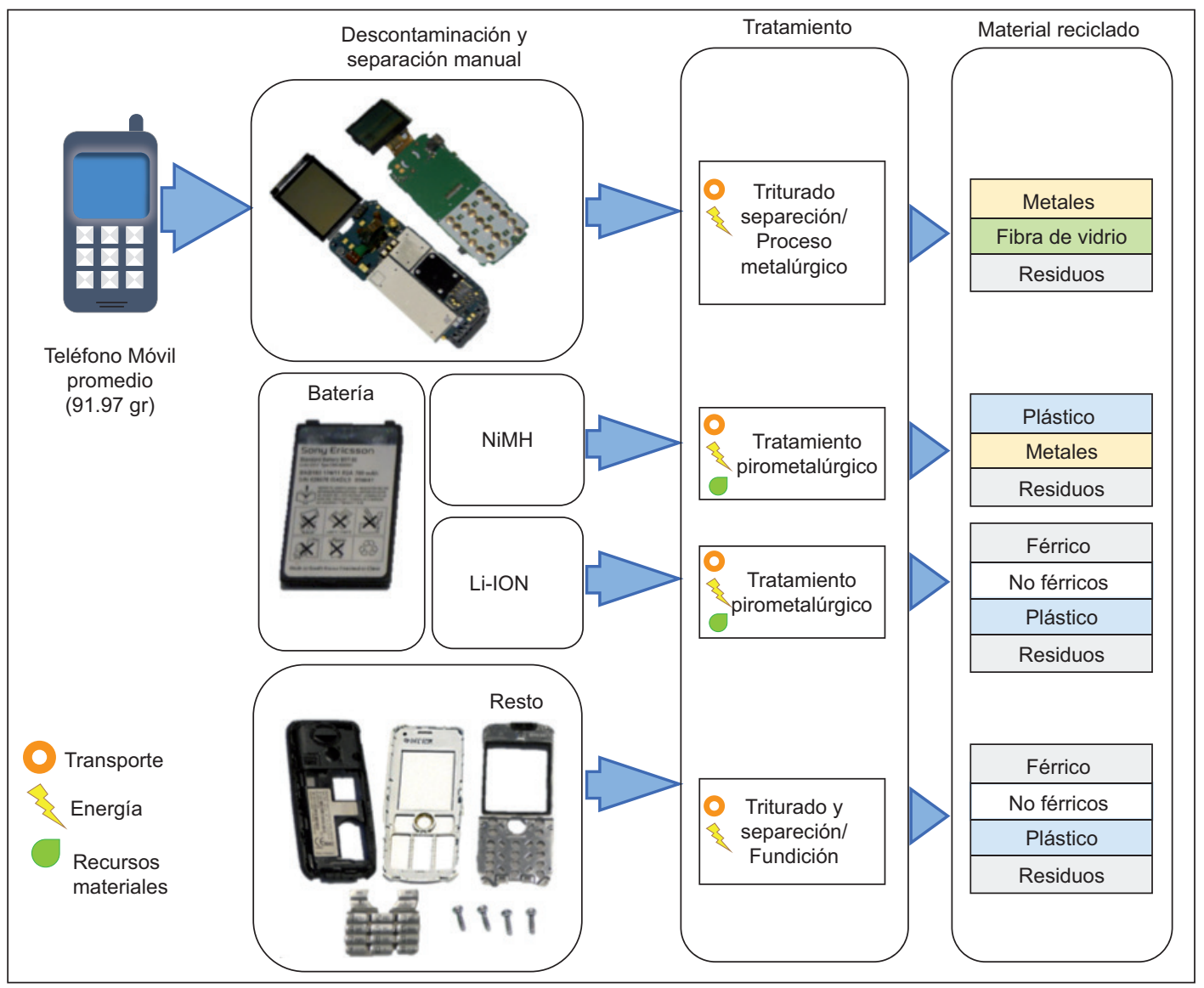

Fig. 2. Proceso de tratamiento del teléfono móvil. Fuente. Elaboración propia

CUADRO X. RECICLADO DE COMPONENTES DEL MÓVIL

\begin{tabular}{|c|c|c|c|}
\hline \multicolumn{2}{|c|}{ Componente } & Entradas requeridas & Material reciclable \\
\hline \multirow{8}{*}{ 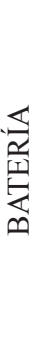 } & Reciclado Li-ion & $0.001 \mathrm{~m}^{3} \mathrm{H}_{2} \mathrm{O}$ & Plásticos mixtos \\
\hline & Pirometalúrgico & $0.8 \mathrm{kw} / \mathrm{h}$ & No férrico \\
\hline & $12.73 \%$ & $0.35 \mathrm{~kg} \mathrm{NaOH}$ & Férrico \\
\hline & & $2240 \mathrm{~kg} / \mathrm{km}$ & Residuos \\
\hline & Reciclado NiMH & $0.00024 \mathrm{~m}^{3} \mathrm{H}_{2} \mathrm{O}$ & Plásticos mixtos \\
\hline & Pirometalúrgico & $0.31 \mathrm{kw} / \mathrm{h}$ & Férrico \\
\hline & $12.96 \%$ & 0.00167 kg carbón & Residuos \\
\hline & & $\begin{array}{l}0.0947 \mathrm{~kg} \text { gas propano/butano } \\
2888 \mathrm{~km}\end{array}$ & \\
\hline \multirow{8}{*}{ 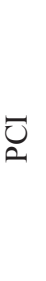 } & Reciclado PCI & $0.04 \mathrm{kw} / \mathrm{h}$ & $\mathrm{Au}$ \\
\hline & $26.9 \%$ & $2420 \mathrm{~kg} / \mathrm{km}$ & $\mathrm{Ag}$ \\
\hline & & & Al \\
\hline & & & $\mathrm{Cu}$ \\
\hline & & & $\mathrm{Ni}$ \\
\hline & & & $\mathrm{Pb}$ \\
\hline & & & Plástico \\
\hline & & & Residuo \\
\hline \multirow{4}{*}{ 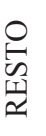 } & Resto del móvil & & Plástico \\
\hline & (carcasas, tornillos, & & Férrico \\
\hline & teclados, etc.) & & No Férrico \\
\hline & $47.41 \%$ & & \\
\hline
\end{tabular}

$\mathrm{PCI}=$ placa de circuito impreso 
CUADRO XI. RECICLADO DE MATERIALES DEL TELÉFONO MÓVIL

\begin{tabular}{ll}
\hline Cargas evitadas & Materiales secundarios \\
\hline Aluminio, primario, en planta & Aluminio reciclado, en planta \\
\hline Cobre, primario, en refinería & Cobre secundario, del reciclaje de RAEE, en refinería \\
\hline $\begin{array}{l}\text { Níquel, primario, a partir de la producción } \\
\text { de metales del grupo del platino }\end{array}$ & Níquel, secundario, del reciclaje de RAEE, en refinería \\
\hline Oro, primario, en refinería & Oro, secundario, en refinería de metales preciosos \\
\hline Plata, almacenamiento regional & Plata, secundario, en refinería de metales preciosos \\
\hline Platino, primario, en refinería & Platino, secundario, en refinería \\
\hline $\begin{array}{l}\text { Plomo, primario, en planta } \\
\text { Zinc, primario, almacenamiento regional }\end{array}$ & Plomo, secundario, del reciclaje de RAEE, en planta \\
\hline Hierro, en planta & Hierro secundario, en planta \\
\hline $\begin{array}{l}\text { Poliestireno (PS) } \\
\text { Policloruro de vinilo (PVC) }\end{array}$ & $\begin{array}{l}\text { Poliestireno del reciclaje de RAEE, } \\
\text { Policlorileno Tereftalato (PET) } \\
\text { Polietileno tereftalato del reciclaje de RAEE, }\end{array}$ \\
\hline
\end{tabular}

RAEE $=$ residuos de aparatos eléctricos y electrónicos

CUADRO XII. CUANTIFICACIÓN DE IMPACTO AMBIENTAL POR ESCENARIO (S)

\begin{tabular}{|c|c|c|c|}
\hline Categoría de Impacto & $\mathrm{S}_{2}$ & $\mathrm{~S}_{1}$ & $\mathrm{~S}_{0}$ \\
\hline Acidificación ( $\mathrm{kg} \mathrm{SO}_{2}$ eq) & 0.2329 & 0.2426 & 0.2530 \\
\hline Eutrofización ( $\mathrm{kg} \mathrm{PO}_{4}$ eq) & 0.0076 & 0.0094 & 0.0113 \\
\hline Calentamiento global $\left(\mathrm{kg} \mathrm{CO}_{2} \mathrm{eq}\right)$ & 22.3995 & 23.0907 & 23.8438 \\
\hline Destrucción de capa de ozono (kg CFC-11 eq) & $8.13 \times 10^{-6}$ & $1.08 \times 10^{-5}$ & $1.35 \times 10^{-5}$ \\
\hline Oxidación fotoquímica ( $\left.\mathrm{kg} \mathrm{C}_{2} \mathrm{H}_{4} \mathrm{eq}\right)$ & 0.0138 & 0.0151 & 0.0164 \\
\hline
\end{tabular}

kg eq. = kilogramos equivalentes, $\mathrm{S}_{0}=$ escenario base ( 18 meses de uso), $\mathrm{S}_{1}=24$ meses de uso, $\mathrm{S}_{2}=30$ meses de uso

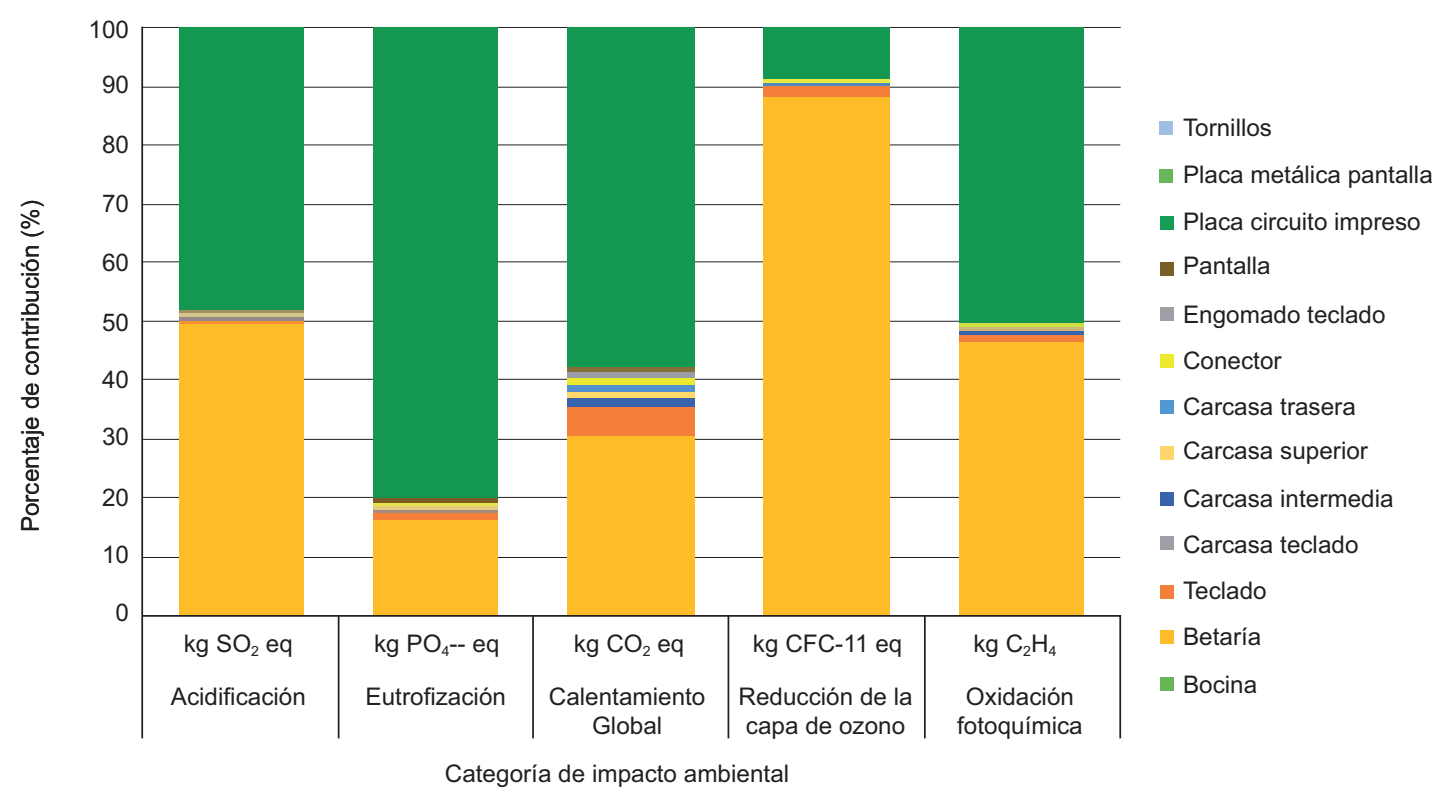

Fig. 3. Evaluación ambiental por componente del móvil promedio 


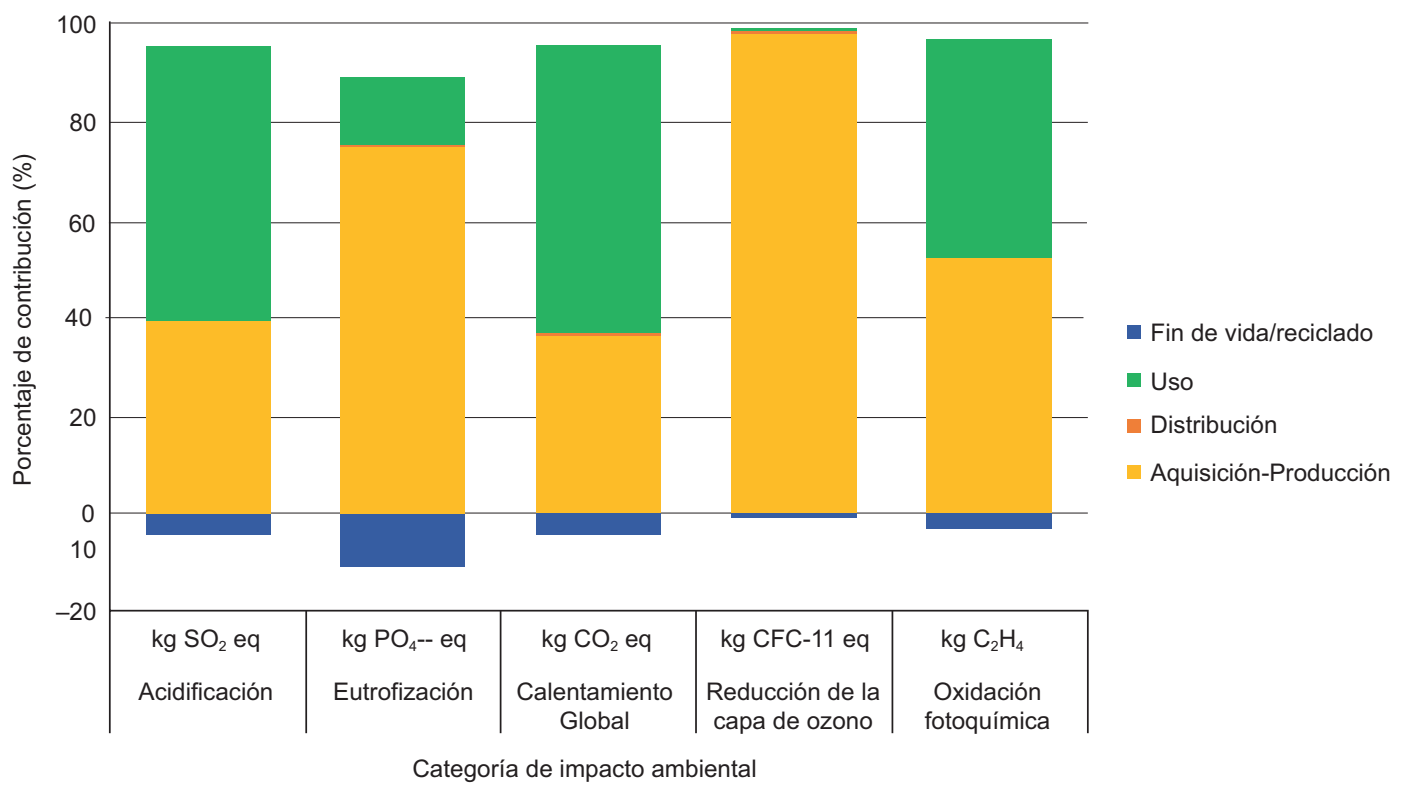

Fig. 4. Evaluación ambiental por etapa de vida del teléfono móvil

mayor uso de recursos. Bento (2016), afirma que la preocupación por los impactos negativos asociados al ciclo de vida de los teléfonos móviles está en relación con los grandes volúmenes de producción y características de corto tiempo de vida.

La figura 3 muestra que la batería y la PCI son los componentes que más contribuyen al impacto ambiental; la primera a la reducción de capa de ozono, y la segunda a la categoría de eutrofización.

La figura 4 muestra el nivel de contribución al impacto ambiental por etapas de ciclo de vida del teléfono móvil, las de mayor impacto son adquisición, producción y uso. En la primera etapa, la categoría de mayor contribución es la reducción de la capa de ozono, asociada a la extracción de materias primas. En la segunda, la categoría de mayor impacto es calentamiento global, asociada a la generación y consumo de la energía eléctrica. Suckling y Lee (2015), afirman que la etapa de fabricación del móvil es la que aporta más emisiones de gases de efecto invernadero. Otro estudio estima que la manufactura del móvil produce $60 \mathrm{~kg}$ de $\mathrm{CO}_{2}$ eq y la etapa de uso en un año produce cerca de $122 \mathrm{~kg}$ de $\mathrm{CO}_{2}$ eq (Velmurugan 2017).

En la figura 5 se compara el nivel de contribución al impacto ambiental por escenario. En todas las categorías se observa una reducción para $\mathrm{S}_{2}$, mostrándose una reducción de casi el $40 \%$ en la categoría de destrucción de la capa de ozono.

En general, se observa que al prolongar el periodo de uso del teléfono móvil se evitan cargas contaminantes, al no producirse más residuos y se disminuye el consumo de materia prima virgen en la producción de nuevos equipos. También se observa que a medida que se extiende el tiempo de vida útil de los teléfonos móviles los potenciales impactos ambientales se reducen en todas las categorías por la disminución en la demanda de materias primas y energía. Esto representa una oportunidad de intervención en la concientización de usuarios sobre el manejo del móvil en relación con las prácticas de cargas del dispositivo, con el propósito que administren los tiempos y momentos de carga, para evitar daños al equipo como consecuencia de la sobrecarga y calentamientos lo cual impacta en la disminución de su vida útil.

Ongondo (2011), explica que la reutilización de estos dispositivos y su composición en términos de masa y volumen representan los productos electrónicos más valiosos que actualmente se encuentran en gran número en el flujo de residuos, por lo que al final de su vida útil son productos de alto valor. Sin embargo, la complejidad de los diseños dificulta los procesos de tratamiento para la recuperación de fracciones. En este sentido Nnorom (2009), menciona que entre las barreras para el reciclaje de RAEE, está la falta de conciencia sobre riesgos de toxicidad y el no usar técnicas correctas en la gestión. Además, Welfens y Seibt (2016) señalan que se requiere de una combinación de varios factores económicos, sociales, institucionales y culturales para una gestión efectiva. 

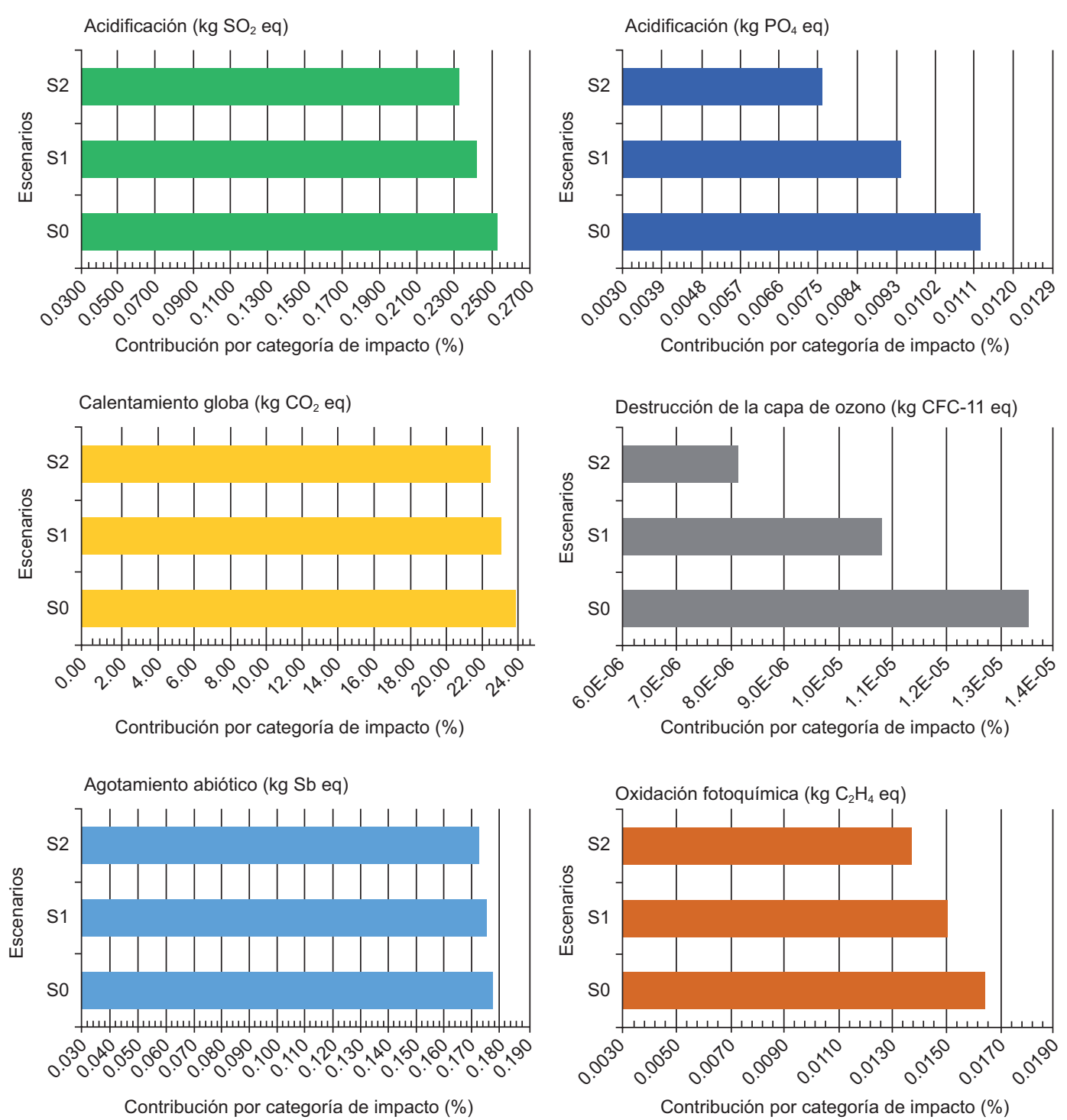

Fig. 5. Evaluación ambiental por escenario

Liao (2013) afirma que el desarrollo de productos respetuosos del ambiente se está convirtiendo en una práctica estándar de producción, a través de iniciativas para el ahorro en el consumo de energía, reducción de emisiones y diseños orientados a promover el uso eficaz de los recursos y el reciclaje.

\section{CONCLUSIONES}

Es importante implementar medidas de gestión de teléfonos móviles al final de su vida útil, teniendo en cuenta las oportunidades y beneficios que representa su reutilización, considerando además, que son los equipos electrónicos de mayor consumo y que más se disponen en el flujo de los RSU. Sin embargo, con el fin de formular estrategias, es necesario tener en cuenta que la posibilidad de reciclar las fracciones recuperables depende de una serie de factores tales como la rentabilidad de los procesos de reciclaje, la existencia de mercados para el material reciclado, volumen, concentración y pureza del material reciclado y la existencia de tecnologías de separación y reciclado e infraestructura.

Los resultados muestran que es importante cuantificar las cargas evitadas por la recuperación de materiales y el aumento del tiempo de vida útil al teléfono móvil, porque se evita la adquisición de nuevos dispositivos y la generación de residuos. Además, se reduce el combustible consumido durante 
la distribución del producto, asimismo disminuye el volumen de dispositivos en desuso que van a tratamiento, lo que significa menor impacto al deterioro de la capa de ozono.

En la gestión de RAEE el reciclaje juega un papel clave porque reduce el impacto ambiental debido a las cargas contaminantes evitadas por los tratamientos, ahorra recursos limitados y la energía usada para su extracción a través de la recuperación de los materiales que se reincorporan a la cadena de producción.

Sin embargo, antes de reciclar es importante considerar la reparación y reutilización del móvil, evitando que se convierta en residuo ya que una cantidad considerable de estos se desechan por razones de moda o estética siendo aún funcionales.

El estudio realizado muestra cómo a través del $\mathrm{ACV}$ es posible identificar los potenciales impactos ambientales y, además, determinar en qué etapa del ciclo de vida del teléfono móvil es posible intervenir para reducir o minimizar estos impactos. De acuerdo con los resultados el escenario de gestión $\mathrm{S}_{2}$ es el que trae mayor beneficio.

\section{AGRADECIMIENTOS}

Los autores agradecen al Programa Iberoamericano de Ciencia y Tecnología para el Desarrollo (CYTED) por la concesión del proyecto de la Red Iberoamericana en Gestión y Aprovechamiento de Residuos (REDIGAR).

\section{REFERENCIAS}

Aleksic S. (2014). Green ICT for sustainability: A holistic approach. In information and communication technology, electronics and microelectronics (MIPRO). Memorias. 37th International Convention on IEEE. Opatija, Croacia. 26 al 30 mayo, 2014. 426-431.

DOI: 10.1109/MIPRO.2014.6859604

Baldé C.P., Wang F., Kuehr R. y Huisman J. (2015) The global e-waste monitor - 2014, United Nations University, IAS - SCYCLE, United Nations University (UNU), Bonn, Alemania, 79 pp.

Barton J. R., Dalley D. y Patel V. S. (1996). Life cycle assessment for waste management. Waste Manage. 16 (1), 35-50.

DOI: 10.1016/S0956-053X(96)00057-8

Bento N. (2016). Calling for change? Innovation, diffusion, and the energy impacts of global mobile telephony. Energy Res. Soc. Sci. 21, 84-100.

DOI: $10.1016 /$ j.erss.2016.06.016
Cleary J. (2009). Life cycle assessments of municipal solid waste management systems: A comparative analysis of selected peer-reviewed literature. Environ. Int. 35 (8), 1256-1266. DOI: 10.1016/j.envint.2009.07.009

Cucchiella F., D’Adamo I., Koh S. L. y Rosa P. (2015). Recycling of WEEEs: An economic assessment of present and future e-waste streams. Renew Sust. Energ. Rev. 51, 263-272.

DOI: $10.1016 /$ j.rser.2015.06.010

Ecoinvent (2008). The life cycle inventory data version 2. Swiss Centre for Life Cycle Inventories [en línea]. http://www.ecoinvent.org/database/olderversions/ecoinvent-version-2/ecoinvent-version-2. html 04/07/2016

Ekvall T., Assefa G., Björklund A., Eriksson O. y Finnveden G. (2007). What life-cycle assessment does and does not do in assessments of waste management. Waste Manage. 27 (8), 989-996. DOI: $10.1016 /$ j.wasman.2007.02.015

Foolmaun R.K. y Ramjeawon T. (2008). Life cycle assessment (LCA) of PET bottles and comparative LCA of three disposal options in Mauritius. Int. J. Environment and Waste Manag. 2, 125-130.

DOI: 10.1504/IJEWM.2008.016997

Guinée J. (2002) Handbook on life cycle assessment. An operational guide to the ISO standards. Kluwer Academic Publishers, Nueva York, EUA, 692 pp.

ITU (2016). World telecommunication/ICT indicators database. Key ICT indicators for developed and developing countries and the world (totals and penetration rates). International Telecommunication Union [en línea]. http://www.itu.int/en/ITU-D/Statistics/Pages/ definitions/regions.aspx 20/09/2016

ISO (2006). ISO 14040. Environmental management. Life cycle assessment. Principles and framework. International Organization for Standardization. Julio 2006 [en línea] https://www.iso.org/standard/37456. $\mathrm{html} 02 / 08 / 2016$

ISO (2006). ISO 14044. Environmental management. Life cycle assessment. Requirements and guidelines. International Organization for Standardization. Julio 2006 [en línea] https://www.iso.org/standard/38498. $\mathrm{html} 02 / 08 / 2016$

Kasper A. C., Bernarders A. M. y Veit H. M. (2011). Characterization and recovery of polymers from mobile phone scrap. Waste Manag. Research. 29, 714. DOI: $10.1177 / 0734242 X 10391528$

Kiddee P., Naidu R. y Wong M. H. (2013). Electronic waste management approaches: An overview. Waste Manag. 33 (5), 1237-1250.

DOI: $10.1016 /$ j.wasman.2013.01.006

Liao C. S., Lo K. R. y Gao C. T. (2013). Sustainable development of electrical and electronic equipment: 
User-driven green design for cell phones. Bus. Strat. Env. 22 (1), 36-48.

DOI: $10.1002 /$ bse. 743

Magaline F. Kuehr R. y Baldé C.P. (2015). eWaste in Latin America Statistical analysis and policy recommendations. GSMA/Institute for the Advanced Study of Sustainability (UNU-IAS) [en línea] https://www. gsma.com/latinamerica/wp-content/uploads/2015/11/ gsma-unu-ewaste2015-eng.pdf 20/09/2016

Mohabuth N. y Miles N. (2005). The recovery of recyclable materials from waste electrical and electronic equipment (WEEE) by using vertical vibration separation. Resour. Conserv. Recy. 45 (1), 60-69.

DOI: $10.1016 /$ j.resconrec.2005.02.001

Nnorom I.C., Ohakwe J. y Osibanjo O. (2009). Survey of willingness of residents to participle in electronic waste recycling in Nigeria -A case study of mobile phone recycling. J. Clean Prod. 17 (18), 1629-1637. DOI: 10.1016/j.jclepro.2009.08.009

Obersteiner G., Binner E., Mostbauer P. y Salhofer S. (2007). Landfill modelling in LCA - a contribution based on empirical data. Waste Manage. 27 (8), S58S74. DOI: 10.1016/j.wasman.2007.02.018

Osibanjo O. y Nnorom I. (2008). Material flows of mobile phones and accessories in Nigeria: Environmental implications and sound end-of-life management options. Environ. Impact Assess. Rev. 28 (2-3), 198-213. DOI: $10.1016 /$ j.eiar.2007.06.002

Ongondo F.O. y Williams I.D. (2011). Greening academia: Use and disposal of mobile phones among university students. Waste Manage. 31 (7), 1617-1634.

DOI: 10.1016/j.wasman.2011.01.031

Peralta G.L. y Fontanas P.M. (2006). E-waste issues and measures in the Philippines. J Mater Cycles Waste Manage. 8 (1), 34-39.

DOI: $10.1007 / \mathrm{s} 10163-005-0142-5$

Song Q., Wang Z., Li J. y Zeng X. (2013). The life cycle assessment of an e-waste treatment enterprise in China. J Mater Cycles Waste Manag. 15 (4), 469-475. DOI: 10.1007/s10163-013-0152-7

Rives J., Rieradevall J. y Gabarrel X. (2010). LCA comparison of container systems in municipal solid waste management. Waste Manage. 30 (6), 949-957.

DOI: 10.1016/j.wasman.2010.01.027

Rosen K. y Meier A. (2001) Energy use of U.S. consumer electronics at the end of the 20th century. En: Energy efficiency in household appliances and lighting. (P. Bertoldi, A. Ricci A. de Almeida, Eds ) Springer, Berlin, Alemania, pp. 256-266.

DOI: 10.1007/978-3-642-56531-1_31
Suckling J. y Lee J. (2015). Redefining scope: the true environmental impact of smartphones?. Int. J. Life Cycle Ass. 20 (8), 1181-1196.

DOI: $10.1007 / \mathrm{s} 11367-015-0909-4$

Schmidt S. y Pahl-Wostl C. (2007) Modelling biowaste flows for life-cycle assessment- calculation of the potential and collected weight of kitchen and garden waste. J. Ind. Ecol. 11 (1), 181-199.

DOI: $10.1162 /$ jiec.2007.1141

SimaPro (2013). SimaPro v8.0.1 PRé Consultants, B.V. Amersfoort, Países Bajos.

Sinha-Khetriwal D., Kraeuchi P. y Schwaninger M. (2005). A comparison of electronic waste recycling in Switzerland and in India. Environ. Impact. Assess. Rev. 25 (5), 492-504. DOI: 10.1016/j.eiar.2005.04.006

Velmurugan M. S. (2017). Sustainable perspectives on energy consumption, EMRF, environment, health and accident risks associated with the use of mobile phones. Renew Sust. Energ. Rev. 67, 192-206.

DOI: 10.1016/j.rser.2016.09.011

Welfens M. J., Nordmann J. y Seibt A. (2016). Drivers and barriers to return and recycling of mobile phones. Case studies of communication and collection campaigns. J. Clean Prod. 132, 108-121. DOI: $10.1016 /$ j.jclepro.2015.11.082

Widmer R., Oswald-Krapf H., Sinha-Ketriwal D., Schnellmann M. y Boni H. (2005). Global perspectives on e-waste. Environ. Impact. Asses. 25 (5), 436-458. DOI: 10.1016/j.eiar.2005.04.001

Winkler J. y Bilitewski B. (2007). Comparative evaluation of life cycle assessment models for solid waste management. Waste Manage. 27 (8), 1021-1031.

DOI: 10.1016/j.wasman.2007.02.023

Yamaguchi H., Tahara K.; Itsubo N. e Inaba A. (2003). A life cycle inventory analysis of cellular phones. Memorias. Proceedings of Eco-design 2003: Third International Sysmposium on Environmentally Conscious Design and Inverse Manufacturing. Tokio, Japón, 8-11 de diciembre, 2003. 445-451.

DOI: 10.1109/ECODIM.2003.1322712

Yla-Mella J., Pongracz E. y Keiski R. L. (2004). Recovery of waste electrical and electronic equipment (WEEE) in Finland. En: Proceedings of the waste minimization and resources use optimization conference (E. Pongrácz, Ed.). University Press, Oulu, Finlandia, pp.83-92

Yu J., Williams E. y Ju M. (2010). Analysis of material and energy consumption of mobile phones in China. Energ. Pol. 38 (8), 4135-4141.

DOI: $10.1016 /$ j.enpol.2010.03.041 